\title{
TERRITORIO, URBANISMO Y CRISIS. UNA MIRADA AL MEDIO VINALOPÓ (ALICANTE) ${ }^{1}$
}

\author{
Xavier Amat Montesinos \\ Dpto. Geografía Humana \\ Universidad de Alicante
}

\section{RESUMEN}

Las claves de la planificación territorial en la Comunidad Valenciana han estado en el protagonismo de la iniciativa municipal y la ausencia de directrices en la escala supralocal. Además, los instrumentos normativos han favorecido la desregulación del suelo y la entrada del capital inmobiliario, con lo que el territorio se ha convertido en el soporte de valor del que administraciones públicas y entidades privadas han obtenido beneficios inmediatos. Multitud de planes y proyectos se han enunciado e iniciado en la región valenciana durante los últimos 15 años, promocionando un modelo de crecimiento que ha alimentado la situación actual de recesión y crisis. La alta indiferencia territorial de estos planes y proyectos, ha provocado la transformación de todo tipo de espacios: litorales, de interior e incluso pueblos de montaña. En el presente documento, se analizan las causas y consecuencias de este fenómeno en la comarca interior alicantina del Medio Vinalopó. Como principales aportaciones, se identifican y caracterizan las actuaciones iniciadas y previstas sobre el territorio desde un punto de vista conjunto de los 11 municipios del área de estudio. Posteriormente, se ofrece un análisis prospectivo del modelo territorial comarcal. Finalmente, se consideran las implicaciones ambientales, económicas y sociales de este modelo de crecimiento que ha conducido a una situación de crisis, tratando de buscar soluciones y oportunidades desde la planificación territorial.

Palabras clave: planificación territorial, urbanismo, crisis, Comunidad Valenciana, Medio Vinalopó.

\section{ABSTRACT}

The key elements of how land planning has developed in the Region of Valencia involve the effects of municipal initiatives and the lack of directives at any higher level. Furthermore, the existing regulatory instruments have led to a deregulation of the land

1 El presente artículo forma parte de la Tesis doctoral en curso del autor, dirigida por D. Vicente Gozálvez Pérez, realizada con el apoyo de una beca FPI de la Generalitat Valenciana en el Dpto. de Geografía Humana de la Universidad de Alicante. 
and the entry of property capital, turning land into a source of value from which public administrations and private enterprises have obtained immediate benefit. In the past 15 years, a huge number of plans and projects have been undertaken or announced in the Region of Valencia that have promoted a model of growth that has fed the current situation of recession and crisis. The indifference with which land is regarded in these plans and projects has led to spaces of all kinds being transformed: on the coast, inland and even in towns and villages in mountainous areas. This paper analyses the causes and consequences of this phenomenon in the inland Medio Vinalopó area of the Alicante province, identifying measures currently being implemented, or scheduled to be implemented, from the joint perspective of 11 municipalities in the area, followed by prospective analysis of the land model applied. Finally, consideration is given to the environmental, economic and social implications of this growth model that has led to a situation of crisis, and suitable land planning solutions and opportunities are sought.

Key words: land planning, urban planning, crisis, Region of Valencia, Medio Vinalopó.

\section{Introducción}

El último periodo de expansión urbanizadora, iniciado en la década de 1990 con una coyuntura económica favorable, se ha basado en la percepción del territorio como soporte de valor para la inversión y la obtención de beneficios en el corto plazo. La permisividad de los instrumentos de planificación territorial y urbanística, unida a factores económicos y financieros favorables a la venta y consumo artificial de suelo, han caracterizado una etapa en la que el crecimiento económico se ha traducido en deterioro ambiental y desorden y despilfarro de territorio (VV.AA, 2009). En una hipótesis compartida por muchos, el boom inmobiliario de los últimos años ha alimentado la situación de crisis actual, cuyas negativas repercusiones han sido más intensas en las áreas de mayor euforia y dinámica constructora.

La Comunidad Valenciana, junto a la de Madrid y al resto de regiones de la fachada mediterránea, ha sido una de las regiones en las que este proceso se ha producido de un modo más intenso y extenso en el territorio ${ }^{2}$, con una extraordinaria concentración temporal (Mata, 2007: 31). Los cambios en la ocupación y uso funcional del territorio han sido inmediatos y explosivos, provocando importantes alteraciones ecológicas y paisajísticas, modificando la lógica económica y productiva de numerosos lugares, y provocando un creciente y dinámico movimiento de respuesta ciudadana, ante la deriva de las políticas de planificación territorial y urbanística. En un fenómeno sin precedentes por su magnitud, las áreas litorales turísticas han sido las que han soportado las mayores transformaciones (en 2009, el $58 \%$ de la costa valenciana es suelo urbano o urbanizable ${ }^{3}$ ). No obstante, en la última fase de este periodo, se observa una expansión hacia las zonas próximas de interior o pequeños pueblos rurales (Fernández, 2006: 25), que ahora se incorporan al proceso urbanizador con una dinámica desenfrenada.

El objetivo del presente trabajo es el de comprobar la irrupción sobre las tierras de interior del fenómeno de la expansión urbanizadora, tratando de profundizar sobre las características de la planificación territorial y urbanística que la han condicionado. Para ello, se ha seleccionado un ámbito interior de la provincia de Alicante, por tratarse de una de las áreas que más ha contribuido al boom inmobiliario durante los últimos años. La comarca del Medio Vinalopó, en el inmediato traspaís de Alicante y Elche, registra una desaforada

2 En el periodo 1996-2006, según datos del Cons. Sup. del Colegio de Arquitectos de España, estas seis autonomías concentran el $65,12 \%$ de los proyectos visados de vivienda en España.

3 Según el informe anual de la organización ecologista Greenpeace, Destrucción a toda costa. 
oferta de suelos para la localización y relocalización de usos residenciales, instalaciones asociadas, usos productivos y vías de comunicación, aprovechando su proximidad a las zonas dinámicas del litoral y su buena disposición desde el punto de vista de las comunicaciones.

Se analizan las actuaciones sobre el territorio iniciadas y previstas en la comarca, avaladas desde los gobiernos locales e incentivadas en multitud de ocasiones por empresas privadas, en procedimientos de difusa y polémica tramitación. Un análisis conjunto de los diferentes planes y proyectos enunciados desde los 11 municipios comarcales permite extraer interesantes reflexiones sobre el futuro territorial deseado por las autoridades locales. Con todo, el desorbitado número y la intensidad de las actuaciones propuestas puede provocar unas secuelas económicas, ambientales y sociales, todavía no advertidas, pero que ya están teniendo unas repercusiones negativas, manifestadas a través de la actual situación de recesión y crisis. Como principales aportaciones, en primer lugar, se identifican y caracterizan las actuaciones iniciadas y previstas sobre el territorio del Medio Vinalopó, posteriormente, se ofrece un análisis prospectivo del modelo territorial comarcal, y finalmente, se consideran las implicaciones económicas, ambientales y sociales de este modelo de desarrollo, tratando de buscar alternativas basadas en la coherencia y coordinación de las políticas territoriales.

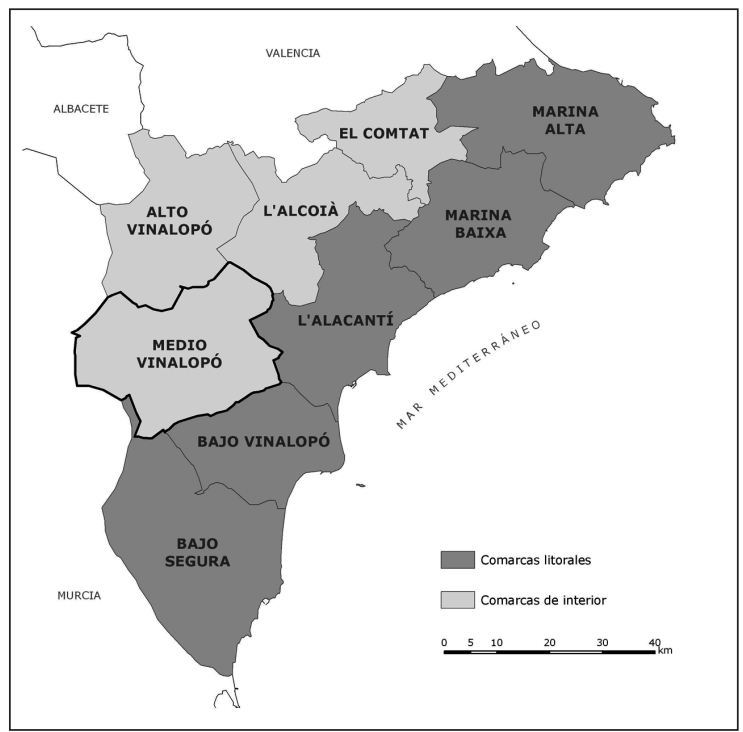

Figura 1. Delimitación y carácter litoral o interior de las comarcas de la provincia de Alicante.

Elaboración propia.

\section{Metodología y fuentes}

Se ha seleccionado el Medio Vinalopó como ámbito de estudio porque sobre esta comarca se identifican los principales elementos que han caracterizado el último periodo de expansión urbanizadora: el salto hacia zonas interiores de los procesos urbanizadores, la existencia de un ambiente generalizado de euforia constructora seguida de una situación de 
recesión y crisis, el protagonismo de las iniciativas locales en la planificación territorial, y la ausencia de unas directrices supramunicipales capaces de orientar y coordinar los crecimientos territoriales y urbanísticos.

Para tratar de validar estas cuestiones, se han identificado, caracterizado, medido en intensidad y proyectado hacia un futuro posible, los planes y proyectos de actuación en el territorio enunciados o iniciados durante la última década. Para ello se ha recurrido a los planes generales vigentes y a las nuevas propuestas urbanísticas, publicadas en el Diario Oficial de la Comunidad Valenciana cuando han superado los trámites administrativos. El objetivo era conocer los parámetros principales de suelo urbano y urbanizable actual y previsto. Otras veces, se han reconocido actuaciones en fase de elaboración o tramitación que todavía no han pasado ningún tipo de filtro público. Los parámetros básicos de estas actuaciones se han definido a partir de la información obtenida en la prensa o a partir de informadores directos (colectivos cívicos, técnicos municipales, etc.), ya que, en la mayoría de las ocasiones, esta información suele ser de difícil acceso, debido a la opacidad estadística en relación con el planeamiento urbanístico en la Comunidad Valenciana (Burriel, 2009a: 36) ${ }^{4}$. Identificadas las previsibles transformaciones y valoradas en conjunto, se establece un hipotético modelo territorial futuro basado en técnicas prospectivas (Lleó, 1979; Gómez, 2008), ubicado en un horizonte temporal en el medio plazo ${ }^{5}$. La confrontación de un escenario futuro con las características del modelo territorial actual pone de manifiesto la magnitud de los cambios propuestos, y advierte sobre sus posibles repercusiones económicas, ambientales y sociales.

Finalmente se establece un conjunto de consideraciones y recomendaciones que abogan por nuevas formas de uso y gestión del territorio a nivel general, y una mayor coordinación y coherencia de las políticas territoriales en el caso concreto del Medio Vinalopó.

\section{Los problemas de la planificación territorial en la Comunidad Valenciana}

La fase de boom inmobiliario entre mediados de los años 1990 y 2007, haciendo uso de la estadística, ha sido especialmente activa en cuanto a urbanización de suelos y construcción de viviendas. En tan sólo una década, en la Comunidad Valenciana se visaron 863.512 proyectos de viviendas (Gaja, 2008) y desaparecieron unos 180 millones de $\mathrm{m}^{2}$ de suelo rústico (Burriel, 2009b) para acoger nuevos usos urbanos. A la producción de suelo residencial, se ha acompañado además una dinámica oferta de suelos industriales y terciarios, y en determinados contextos se ha generado una intensa artificialización de suelo por la implantación de infraestructuras de transporte. El último periodo de expansión urbanizadora, que incluye urbanización de suelos residenciales, industriales, terciarios y vías de comunicación, ha provocado unos cambios de tal magnitud que cuestionan la capacidad de planificar y gestionar el territorio por parte de las administraciones competentes y de los instrumentos de ordenación territorial. Desde diversos análisis (Mata, 2007; Gaja, 2008), se señala que una de las causas de este fenómeno ha sido la permisividad de la Ley de Suelo de ámbito estatal de 1998. La denominada ley del todo urbanizable, que introdujo como novedad la posibilidad de recalificar suelos rústicos sin demasiadas restricciones, así como un conjunto de medidas encaminadas a flexibilizar el mercado del suelo. En la Comunidad Valenciana se identifican además otros problemas vinculados con los instrumentos y mecanismos de planificación, que se resumen en una ausencia de directrices adecuadas en la escala supramunicipal o en el excesivo protagonismo del planeamiento municipal.

4 Desde 1998 no se publica la relación de planes generales municipales aprobados, ni su número anual, ni un resumen de sus parámetros básicos (Burniel, 2009a:36).

5 Considerando que el periodo normal de vigencia de los planes territoriales y urbanísticos es de 10 o 12 años, se establece como horizonte el periodo 2020-2025. 


\subsection{La planificación territorial en la Comunidad Valenciana}

La Ley valenciana Reguladora de la Actividad Urbanística (LRAU) de 1994, supuso el punto de partida del último periodo de expansión urbanizadora en la Comunidad. Sin llegar a desarrollarse reglamentariamente, hasta que perdió su vigencia en 2006 en sustitución de la Ley Urbanística Valenciana, en pocos años la LRAU introdujo cambios sustanciales sobre los modos de intervenir sobre el suelo. La introducción de la figura del agente urbanizador y de un nuevo tipo de desarrollo de proyectos urbanísticos más rápidos y flexibles, los denominados Programas de Actuación Urbanística (PAI), modificaron los procesos urbanizadores, posibilitando la recalificación de suelos y la entrada de capital inmobiliario. En definitiva, permitiendo desbloquear e incentivar la intervención sobre el suelo de la iniciativa privada.

Por su parte, la Ley de Ordenación del Territorio (LOT) de 1989, es decir, el instrumento que en apariencia debería regular y coordinar los crecimientos urbanísticos desde un punto de vista estratégico, apenas tuvo repercusión, y, ni siquiera el Plan de Ordenación Territorial, ni los planes de acción (PAT) sectoriales e integrados (propuestos como instrumentos eficaces para planificación y gestión territorial regional y subregional), fueron desarrollados en 15 años. Durante este periodo, en ausencia de unas directrices de planificación, los planes y proyectos urbanísticos propuestos desde los municipios y por los agentes urbanizadores, fueron los verdaderos encargados de «planificar» el territorio. En 2004, la Ley de Ordenación del Territorio y Protección del Paisaje (LOTPP) sustituye a la LOT como instrumento normativo que debe regular el marco de la ordenación del territorio valenciano, e introduce un nuevo instrumento de ordenación global, la Estrategia Territorial de la Comunidad Valenciana (ETV), que, a efectos prácticos, debería establecer las directrices básicas de planificación para los próximos años. La ETV vuelve a incorporar los PAT como herramientas para lograr una planificación efectiva, no obstante, más de un lustro después de su enunciado, la ETV y los distintos PAT sectoriales se encuentran todavía dando sus pasos iniciales, y además, lejos de proponer una planificación y gestión territorial sólida y coherente, en su normativa predominan las meras recomendaciones y las directrices indicativas, y cuando son vinculantes se trata de criterios muy generales o ambiguos (Burriel, 2009b).

Con esto, lo que finalmente se produce es una ausencia de planificación durante los últimos años sobre las escalas en las que se debería tener una visión estratégica y coordinada del territorio, es decir, las escalas regional y supramunicipal. No existen criterios directores sobre territorios amplios, en los que es necesario adecuar elementos tan básicos como las infraestructuras de comunicación y los usos de suelo de interés general. A diferencia de lo que ha ocurrido en otras regiones, en la valenciana no existen instrumentos reales de planificación más allá de la escala local, y es la normativa urbanística municipal la que está imponiéndose en la planificación territorial, pese a la irracionalidad e incongruencias que muchas veces plantea. En definitiva, lo que se detecta es una actitud permisiva del gobierno regional, que ha dimitido voluntariamente de sus obligaciones de control y coherencia a la hora de aprobar propuestas insensatas desde diferentes municipios, y de sus capacidades de coordinación y de elaboración de planes territoriales de ámbito supramunicipal (Romero, 2009: 240).

\subsection{La lógica municipal de la planificación}

La planificación territorial en la Comunidad Valenciana responde por tanto a una lógica local, legitimada a partir de la importancia que se otorga al planeamiento urbanístico municipal, en ausencia de planes de carácter supramunicipal. El territorio se ha ido transformando a partir de intereses de corporaciones municipales y de la influencia que sobre éstas ejerce el capital inmobiliario. Las consecuencias, en un contexto en el que urbanización y construcción 
se han asociado a crecimiento económico, han sido devastadoras con el territorio. La euforia urbanística se extiende, en primer lugar, por los espacios litorales, y durante los últimos años se introduce hacia zonas de interior. Proyectos como Marina d'Or, con una superficie de 19 millones de $\mathrm{m}^{2}$, miles de viviendas y 3 campos de golf, el Manhattan de Cullera, que reclasificaría 8,3 millones de $\mathrm{m}^{2}$ para dar cabida a 14.000 viviendas, el PAI Nou Mil $\cdot l e n i$ de Catarroja (11.500 viviendas) o el Plan Rabassa en Alicante (16.000 viviendas), han sido el espejo en el que se han mirado la mayoría de municipios. Sólo así se entiende que pequeños pueblos rurales, relativamente próximos a las zonas costeras y animados por el auge inmobiliario, se hayan incorporado a este fenómeno promoviendo grandes proyectos de actuación sobre el territorio. Así por ejemplo, en la provincia de Alicante se detectan planes como el PAI de Parcent (1.800 viviendas), el PAI turístico-residencial de Orxeta, con 2,8 millones de $\mathrm{m}^{2}$ y un campo de golf, o las grandes recalificaciones de Sella y Relleu con la mismo finalidad (Giménez y Díez, 2009). En cualquier caso, esto es sólo la punta del iceberg de un fenómeno que se ha extendido con intensidad hacia la mayoría de espacios del interior alicantino.

\section{El ejemplo del Medio Vinalopó}

Las consecuencias de una planificación excesivamente municipalista y carente de unas directrices adecuadas en el ámbito supramunicipal, se hacen más visibles cuando se toma como referencia una escala territorial amplia. Si se analiza la situación por separado de municipios próximos y siguiendo una lógica local, probablemente las propuestas de planeamiento y proyección sobre el territorio podrán parecer correctas y adecuadas a las posibilidades de un municipio. No obstante, si se realiza el ejercicio de sumar las propuestas de varios municipios de un mismo ámbito (Mata, 2007; Burriel, 2009a), la dimensión de los resultados obtenidos puede poner en tela de juicio la coherencia y viabilidad de la planificación en esa zona.

En la comarca del Medio Vinalopó, como consecuencia de la expansión hacia tierras interiores de los procesos urbanizadores en la provincia de Alicante, y en un ambiente generalizado de euforia constructora por parte de los municipios, durante la última década se observa un intenso y dinámico proceso de enunciado de planes y proyectos en el territorio. Sobre la justificación de atraer inversiones, diversificar y dinamizar la actividad económica o generar puestos de trabajo, los municipios proponen o inician grandes proyectos sobre suelo residencial e industrial. Sin unos límites establecidos, en ausencia de una coordinación con municipios vecinos y sin unas directrices claras por parte del gobierno autonómico, la mayoría de municipios están proyectando extensas superficies residenciales, en algunos casos acompañadas por campos de golf, y nuevos lotes de suelo industrial, que no tienen en cuenta ni la demanda ni las posibilidades de éxito de las nuevas actuaciones. Ni mucho menos la desmesurada oferta que se genera si se realiza la suma de todos los municipios. El simple ejercicio de identificar, medir en intensidad y valorar los planes y proyectos propuestos en el conjunto comarcal, puede ofrecer algunas consideraciones respecto a la amplitud y desmesura de territorio que se pretende transformar.

\subsection{Los excesos del urbanismo residencial}

Si se considera el suelo urbanizable residencial en los planes generales vigentes, así como el suelo propuesto en los planes generales en elaboración o en los PAIs, se contabiliza una oferta de nuevo suelo residencial próxima a los 35,4 millones de $\mathrm{m}^{2}$, y un número de viviendas de nueva edificación de 41.831. Esto supondría un incremento de 4,1 en el suelo residencial y de 1,5 en el número de viviendas en la comarca, introduciendo además significativas variaciones en su distribución intermunicipal. 


\begin{tabular}{|c|c|c|c|c|c|c|c|c|}
\hline \multirow[b]{2}{*}{ Municipio } & \multicolumn{2}{|c|}{$\begin{array}{c}\text { Situación actual, } 2009 \\
\text { (A) }\end{array}$} & \multicolumn{2}{|c|}{$\begin{array}{c}\text { Incremento propuesto } \\
\text { (B) }\end{array}$} & \multicolumn{4}{|c|}{ Situación prevista, 2020-2025 } \\
\hline & \begin{tabular}{|c|} 
Suelo \\
urbano \\
residencial \\
(en \\
millones \\
de $^{2}$ ) \\
\end{tabular} & $\begin{array}{c}\text { Número } \\
\text { de } \\
\text { viviendas }\end{array}$ & $\begin{array}{c}\text { Suelo } \\
\text { urbanizable } \\
\text { residencial } \\
(\text { en millones } \\
{\left.\text { de } \mathbf{~ m}^{2}\right)}\end{array}$ & $\begin{array}{c}\text { Número } \\
\text { de } \\
\text { viviendas }\end{array}$ & $\begin{array}{c}\text { Suelo } \\
\text { urbano } \\
\text { residencial } \\
(\text { en millones } \\
{\left.\text { de } \mathbf{~ m}^{2}\right)}\end{array}$ & $\begin{array}{l}(\mathbf{A}+\mathbf{B}) \\
/(\mathbf{A})\end{array}$ & $\begin{array}{c}\text { Número } \\
\text { de } \\
\text { viviendas }\end{array}$ & $\begin{array}{c}(\mathbf{A}+\mathbf{B}) \\
/(\mathbf{A})\end{array}$ \\
\hline Algueña & 0,20 & 1.014 & 0,63 & 625 & 0,84 & 4,1 & 1.639 & 1,6 \\
\hline Aspe & 0,96 & 8.050 & 2,49 & 1.500 & 3,45 & 3,6 & 9.550 & 1,2 \\
\hline Elda & 3,00 & 27.507 & 6,32 & 6.500 & 9,32 & 3,1 & 34.007 & 1,2 \\
\hline $\begin{array}{l}\text { Hondón de } \\
\text { las Nieves }\end{array}$ & 0,37 & 2.034 & 4,25 & 4.583 & 4,63 & 12,5 & 6.617 & 3,3 \\
\hline $\begin{array}{l}\text { Hondón de } \\
\text { los Frailes }\end{array}$ & 0,21 & 1.061 & 2,00 & 6.145 & 2,21 & 10,4 & 7.206 & 6,8 \\
\hline $\begin{array}{l}\text { Monforte } \\
\text { del Cid }\end{array}$ & 1,21 & 5.258 & 5,99 & 3.412 & 7,19 & 6,0 & 8.670 & 1,7 \\
\hline Monóvar & 1,12 & 6.822 & 8,03 & 13.818 & 9,15 & 8,2 & 20.640 & 3,0 \\
\hline Novelda & 1,63 & 13.506 & 1,17 & 2.500 & 2,80 & 1,7 & 16.006 & 1,2 \\
\hline Petrer & 1,58 & 16.431 & 3,04 & 465 & 4,62 & 2,9 & 16.896 & 1,0 \\
\hline Pinoso & 0,97 & 4.445 & 0,59 & 1.000 & 1,56 & 1,6 & 5.445 & 1,2 \\
\hline La Romana & 0,29 & 1.711 & 0,87 & 1.283 & 1,16 & 4,0 & 2.994 & 1,8 \\
\hline $\begin{array}{l}\text { Medio } \\
\text { Vinalopó }\end{array}$ & 11,54 & 87.839 & 35,39 & 41.831 & 46,93 & 4,1 & 129.670 & 1,5 \\
\hline
\end{tabular}

Figura 2. Previsión de suelo residencial y viviendas.

Elaboración propia.

El crecimiento previsto resulta especialmente significativo en municipios como Monforte del Cid. El plan general, vigente desde noviembre de 2008, prevé cinco nuevos sectores residenciales (además del suelo previsto junto al núcleo urbano consolidado), que introducirían unos 6 millones de $\mathrm{m}^{2}$ de suelo residencial y 3.412 viviendas. La proximidad de su término municipal respecto a Alicante y Elche, así como a las zonas turísticas del litoral, y la accesibilidad desde las principales vías de comunicación, avala una oferta residencial que actualmente es la más dinámica y efectiva de la comarca, con los sectores ya en funcionamiento de Alenda Golf y Montecid. En Monóvar, las actuaciones propuestas a partir de PAIs o de un nuevo plan general que no consigue ser aprobado, podrían introducir hasta 13.818 viviendas en 8,03 millones de $\mathrm{m}^{2}$ repartidos en 9 sectores residenciales. De este modo, el parque de viviendas de la ciudad podría triplicarse en los próximos años.

A diferencia de estos municipios, las ciudades con una trayectoria urbanística más amplia, también han realizado sus propuestas residenciales aunque con un nivel de transformación inferior respecto a los suelos residenciales preexistentes. Elda, Petrer, Novelda y Aspe, con los proyectos prácticamente descartados de Las Cañadas-El Tite, El Palomaret, Serreta Golf y Tatiana World respectivamente, apuntan a la implantación de urbanizaciones diferenciadas de los núcleos tradicionales, donde el elemento más destacado es la extensión superficial de las mismas (más de 6 millones de $\mathrm{m}^{2}$ en el caso de Elda). 


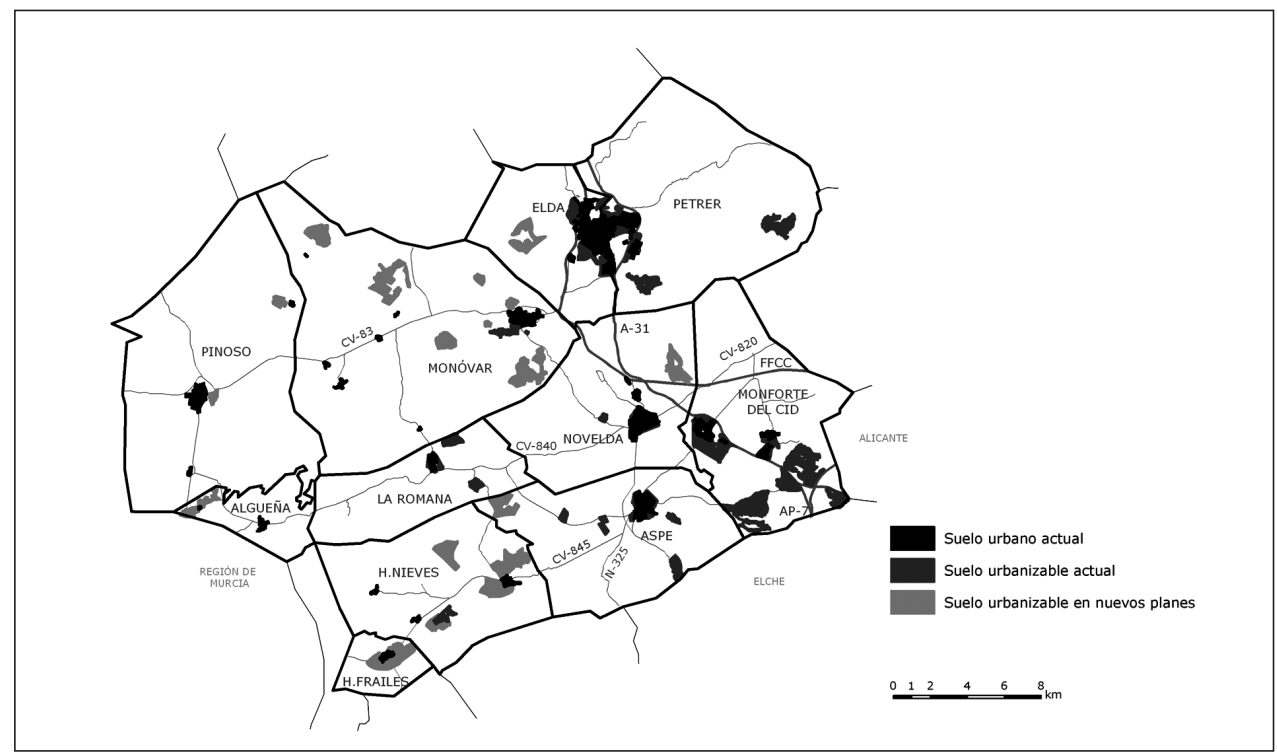

FIGURA 3. Suelo residencial actual y propuesto, 2009.

Fuente: Planes Generales municipales, DOGV y prensa. Elaboración propia.

Con todo, las mayores transformaciones se prevén precisamente sobre los pueblos rurales de la comarca, aquéllos que apuestan decididamente por la venta de suelo como medio para obtener ingresos municipales y animar su actividad productiva a partir de la construcción y servicios derivados de la misma. En este sentido, La Romana ha sido el pueblo pionero en la oferta e inicio de nuevos proyectos residenciales. Con un plan general aprobado en enero de 2006, en pleno auge del sector inmobiliario, se iniciaron las obras de la urbanización Velaire, con una previsión de 689 viviendas. En conjunto, La Romana pretende multiplicar por 4 el suelo residencial y por 1,8 el número de viviendas. De manera similar plantea su crecimiento Algueña con los proyectos Mirador y Laderas de la Solana. Pero especialmente llamativas resultan las intenciones urbanísticas de Hondón de las Nieves y Hondón de los Frailes, con proyectos que multiplicarían el suelo residencial por 12,5 y 10,4 respectivamente (es decir, un núcleo urbano más de diez veces superior al actual), y una previsión de 4.583 y 6.145 nuevas viviendas.

\subsection{La desmesurada oferta de campos de golf}

La oferta residencial viene muchas veces acompañada por instalaciones complementarías que otorgan un valor añadido a determinadas promociones. Así, los campos de golf se han convertido en la justificación más utilizada para avanzar (urbanísticamente) sobre los municipios del interior alicantino ${ }^{6}$, y solamente en el Medio Vinalopó se han propuesto o iniciado hasta 10 campos de golf vinculados a operaciones residenciales.

6 Puede consultarse el artículo de Díaz y Lourés (2008). Navarro y Ortuño (2008) han profundizado sobre estas cuestiones en el proyecto de investigación «Impacto territorial de los campos de golf y operaciones asociadas en el Levante español», encargado al Dpto. Geografía Humana de la Universidad de Alicante. 


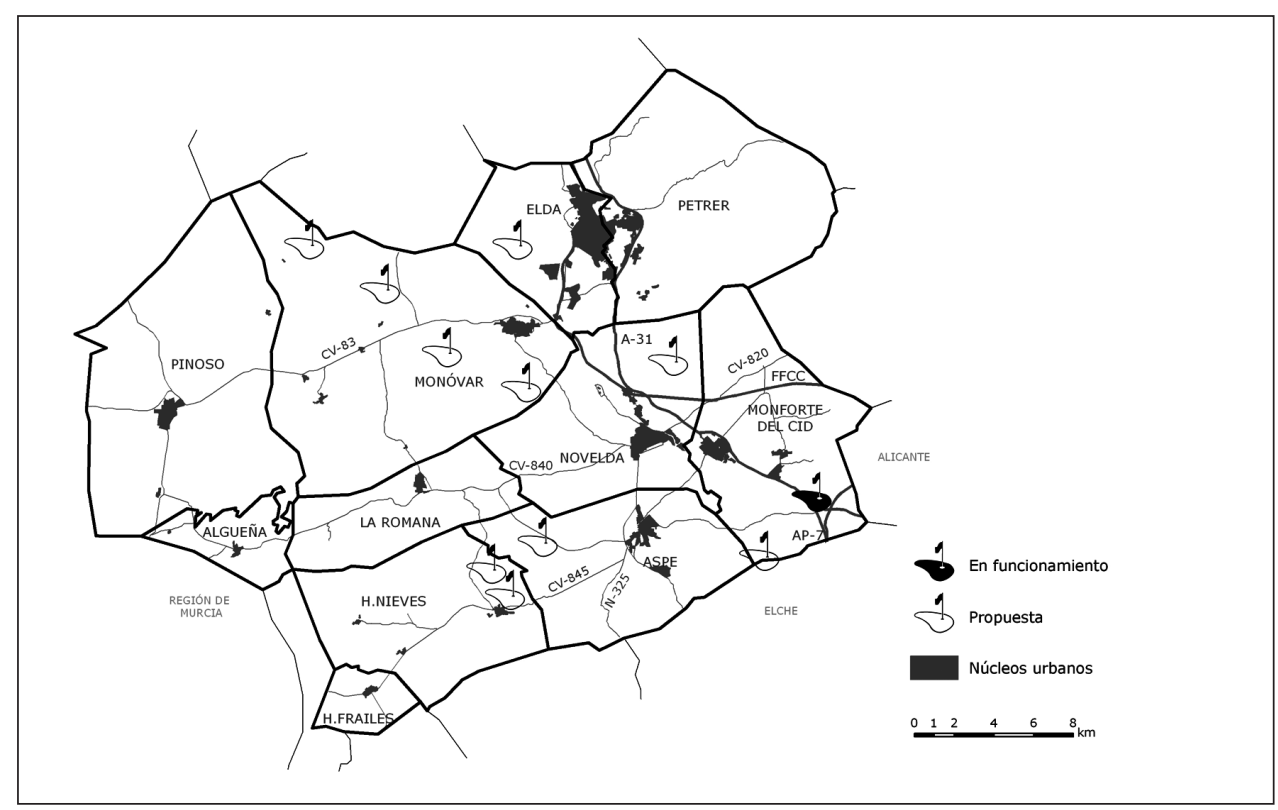

Figura 4. Campos de golf actuales y propuestos, 2009.

Fuente: Planes Generales municipales, DOGV y prensa. Elaboración propia.

La dinámica de la oferta responde en cierto modo a la lógica de la oferta de nuevos suelos residenciales. Habitualmente se recurre a los campos de golf como reclamo publicitario para la venta de residencias anejas al propio campo y aquí, el principal inconveniente es la elevada demanda de agua limpia y suelo, que compite con los usos de suelo preexistentes (Rico y Hernández, 2008: 101). Los campos de golf previstos se localizan preferentemente junto a los grandes proyectos residenciales de Monóvar ( $L a$ Boticaria, Mayorazgos, El Secanet y Betíes), Hondón de las Nieves (Finca la Marquesa y El Rincón) y Monforte del Cid (Font del Llop, además del ya existente campo de Alenda Golf). También Elda, Novelda y Aspe incluyen un campo de golf en las urbanizaciones que proponen. El argumento más empleado para justificar esta desmesurada oferta se encuentra en la necesidad de generar puestos de empleo y orientar la base productiva local hacia el sector turístico. Pero la ausencia de análisis rigurosos por parte de los ayuntamientos y promotores al respecto de la verdadera repercusión de esta actividad, así como la elevada oferta que se produce para una misma zona, parece validar la hipótesis de que los nuevos campos de golf tienen como principal objetivo justificar una importante oferta de suelo residencial y nuevas viviendas.

\subsection{La introducción de nuevas superficies industriales}

Las actuaciones en suelo industrial resultan igualmente extraordinarias, contabilizando hasta 11 proyectos y una importante reserva de suelo destinada a la producción de energía eólica. En conjunto, se prevén 8,66 millones de $\mathrm{m}^{2}$ de suelo industrial que multiplicaría por 3,7 las superficies existentes. 


\begin{tabular}{|c|c|c|c|c|}
\hline \multirow[b]{2}{*}{ Municipios } & \multirow{2}{*}{$\begin{array}{c}\text { Situación actual } \\
2009(\mathrm{~A})\end{array}$} & \multirow{2}{*}{\begin{tabular}{|c|}
$\begin{array}{c}\text { Incremento } \\
\text { propuesto (B) }\end{array}$ \\
$\begin{array}{l}\text { Suelo urbanizable } \\
\text { industrial }(\mathrm{en} \\
\text { millones de } \mathbf{~ m}^{2} \text { ) }\end{array}$ \\
\end{tabular}} & \multicolumn{2}{|c|}{ Situación prevista, 2020-2025 } \\
\hline & & & $\begin{array}{c}\text { Suelo urbano } \\
\text { industrial (en } \\
\text { millones de } \mathbf{m}^{2} \text { ) }\end{array}$ & $(\mathbf{A}+\mathbf{B}) /(\mathbf{A})$ \\
\hline Algueña & - & 0,07 & 0,07 & - \\
\hline Aspe & 0,51 & 1,40 & 1,91 & 3,7 \\
\hline Elda & 1,25 & 0,11 & 1,36 & 1,1 \\
\hline Hondón de las Nieves & - & 0,21 & 0,21 & - \\
\hline Hondón de los Frailes & - & - & - & - \\
\hline Monforte del Cid & 0,36 & 1,99 & 2,35 & 6,5 \\
\hline Monóvar & 0,02 & 0,33 & 0,35 & 20,1 \\
\hline Novelda & 0,58 & 2,26 & 2,84 & 4,9 \\
\hline Petrer & 0,29 & 1,00 & 1,28 & 4,5 \\
\hline Pinoso & 0,23 & 1,00 & 1,23 & 5,4 \\
\hline La Romana & 0,02 & 0,29 & 0,31 & 16,1 \\
\hline Medio Vinalopó & 3,24 & 8,66 & 11,90 & 3,7 \\
\hline
\end{tabular}

FIgURA 5. Previsión de suelo industrial.

Elaboración propia.

A nivel municipal, cabría destacar las ambiciosas iniciativas de Novelda, Monforte del Cid y Aspe. El polígono industrial El Pla de Novelda, avalado por las principales empresas marmoleras de la ciudad, supondría la transformación de 1,6 millones de $\mathrm{m}^{2}$ junto al eje de comunicaciones que supone la autovía y ferrocarril Madrid-Alicante. Próximas a esta zona pero en término municipal de Monforte del Cid, se desarrollan las áreas industriales de Las Norias y Walaig, con 1,5 y 0,8 millones de $\mathrm{m}^{2}$. Por tanto, en un radio no superior a los $10 \mathrm{~km}$, en un futuro habrá una concentración de cerca de 4 millones de $\mathrm{m}^{2}$ de suelo industrial, a los que cabe incorporar además los 0,5 millones de $\mathrm{m}^{2}$ que Aspe prevé con el desarrollo del sector Aljau.

A menor escala, Petrer, Pinoso, Monóvar, La Romana y Algueña han iniciado la construcción de nuevas zonas industriales. En el caso de Petrer, habría que considerar además la superficie incorporada por el Plan Eólico Valenciano (2001). Las sierras del Caballo y de Castalla, deberían acoger sendos parques eólicos en el ámbito de la Zona 15 del plan (donde además, se incluirían los parques de Fontanella, Cenezasa, Alt de la Creu y Argueña en los municipios de Bañeres, Biar, Onil, Castalla y Sax), cuyo potencial de referencia sería de 125 MW a partir de la instalación de 200 aerogeneradores.

En conjunto, la abultada oferta de nuevos suelos industriales se contradice con la dinámica propia de la actividad industrial en la comarca, en un claro proceso de declive, y con la aparente reorientación de algunos municipios hacia el denominado turismo residencial y al sector servicios. Pese al interés por parte de las administraciones locales y la positiva consideración social hacia las zonas industriales, la capacidad de suelo ofertada se intuye excesiva ante la debilidad de la demanda en estos momentos. La creación de grandes lotes de suelo no se corresponde con las perspectivas de implantación por parte de las empresas, y ello puede crear un efecto perverso sobre los propios municipios, ya que tendrán que asumir unos importantes costes de urbanización y seguramente no tendrán la capacidad de atraer las inversiones inicialmente previstas. 


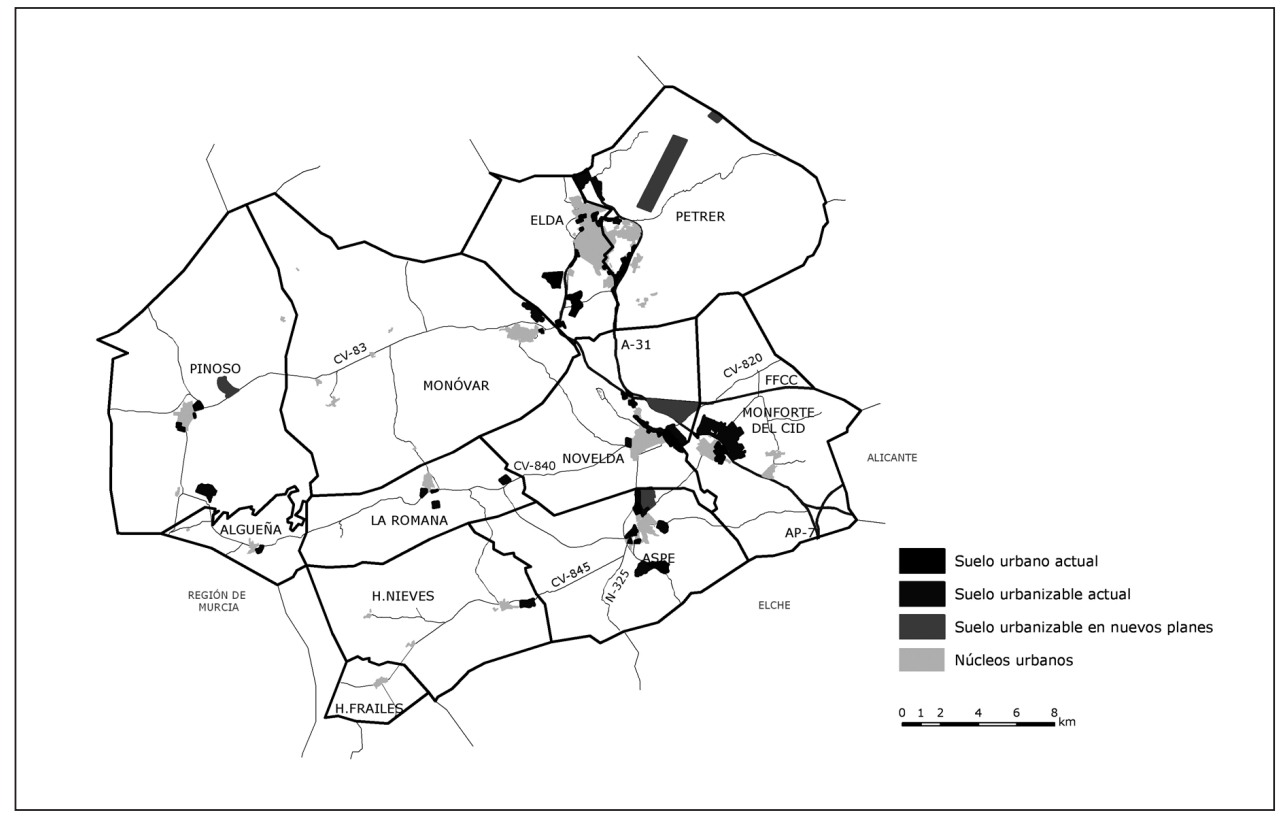

FIgURA 6. Suelo industrial actual y propuesto, 2009.

Fuente: Planes Generales municipales, DOGV, Plan Eólico Valenciano y prensa. Elaboración propia.

\section{4. ¿Qué modelo territorial para el futuro?}

Pero es la definición y diferenciación del modelo territorial actual y su imagen en un futuro a medio plazo, lo que verdaderamente pone de relieve las transformaciones proyectadas sobre el territorio. La combinación de todos los planes y proyectos propuestos y su simulación en una imagen futura, cuestiona el modelo de desarrollo y cultura territorial vigente. Con un mínimo de rigor y sensatez, podrá concluirse que la magnitud de las transformaciones previstas sobrepasa las posibilidades y necesidades de desarrollo para el Medio Vinalopó.

Para observar las principales transformaciones, se ha simulado una posible evolución del modelo territorial, tomando como referencia la situación actual, y otra hipotética situación ubicada en el horizonte 2020-2025, plazo normal de vigencia de los planes y proyectos propuestos. El objetivo es obtener una imagen de futuro del territorio, aplicando herramientas propias de la ordenación territorial como la prospectiva y el recurso de los escenarios. Con todo, suponiendo que se ejecutan todas las actuaciones enunciadas desde la escala local, así como diferentes planes de ámbito nacional y autonómico (infraestructuras y espacios protegidos), se advierten las principales variaciones que sufrirá el modelo territorial en los próximos años. Se trata de las transformaciones que afectan a los elementos estructurantes y más fácilmente representables del modelo territorial (Orea, 2008: 46), por lo que se han considerado los usos primarios del territorio, los asentamientos poblacionales y los canales de conexión.

Entendiendo por usos primarios del territorio la función principal a la que se destina el suelo desde el punto de vista de la actividad humana, el aspecto más destacado es la 


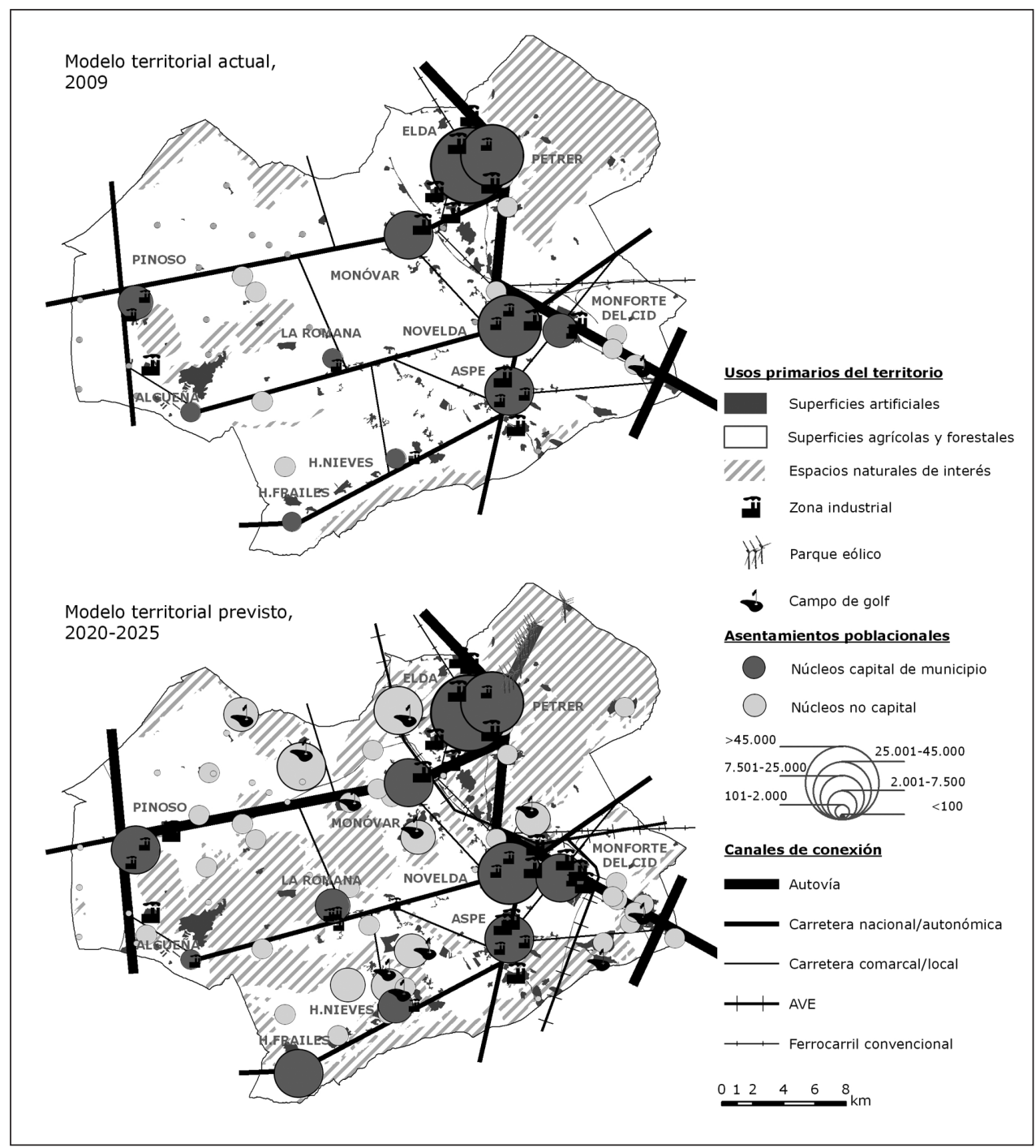

Figura 7. Modelo territorial del Medio Vinalopó

Elaboración propia.

evolución que seguirían las superficies artificiales ${ }^{7}$. Estas superficies, que en la actualidad ocupan unas 5.518 hectáreas, podrían experimentar un crecimiento hasta situarse en las 9.200 hectáreas, debido fundamentalmente al incremento de los suelos residenciales e industriales. Las principales conversiones de suelo se producirían a costa de las superficies agrícolas,

7 Noción adoptada por el proyecto CORINE Land Cover (puede consultarse el informe del Observatorio de la Sostenibilidad en España, 2006), para definir y agrupar al conjunto de zonas urbanas, zonas industriales, comerciales y de transporte, zonas de extracción minera, vertederos y de construcción, y zonas verdes artificiales. 
que experimentarían un fuerte proceso de fragmentación por el impacto provocado por un modelo de urbanización dispersa y extensiva, así como la desarticulación generada por el trazado de las nuevas infraestructuras que conectan las nuevas piezas de suelo urbano en el territorio. Frente a esto, una aparente política de protección de espacios de interés natural que se inicia durante los últimos años, podría contribuir a la salvaguarda de los principales terrenos forestales y agroforestales de la comarca. Si bien las decisiones proteccionistas continúan siendo un hecho excepcional y sin un verdadero carácter estructurante de los espacios naturales de interés con las zonas urbanas.

Desde el punto de vista funcional, destaca la localización y relocalización de los núcleos urbanos y de las áreas industriales, así como la irrupción de nuevos elementos como los campos de golf y los espacios ocupados por parques eólicos. Efectivamente, los núcleos urbanos experimentarían una fluctuación respecto a la trayectoria urbanística de la comarca. Las ciudades de tamaño medio, caso de Elda, Petrer, Novelda y Aspe, que han basado su desarrollo urbano en un modelo de ciudad compacto, perderían su influencia a favor de una multitud de núcleos que aparecerían segregados y aislados en el territorio. La dispersión urbana se materializaría especialmente en las ciudades de tamaño inferior y en los pueblos rurales, en un futuro zonas de vocación eminentemente residencial. Por otra parte, el desarrollo de nuevas áreas industriales reforzaría el carácter industrial de la mayoría de municipios, pues, a excepción de Hondón de los Frailes, todos han proyectado desarrollos más o menos intensos. En este sentido, cabría señalar la concentración de grandes áreas industriales en torno a Novelda, Monforte del Cid y Aspe, aprovechando la proximidad a los principales ejes de comunicaciones. En otros casos, como complemento o alternativa al polígono industrial, los campos de golf se plantean como áreas de atracción económica, con un importante desarrollo en los enclaves más destacados desde el punto de vista ambiental y paisajístico de la comarca.

Por otra parte, una cuestión que suele preocupar es la relativa a los incrementos demográficos y a la distribución futura de la población. Considerando la magnitud de las propuestas residenciales, la nota destacada es el crecimiento explosivo planeado en pequeños núcleos y su dispersión extensiva sobre el territorio. Mientras que los núcleos urbanos tradicionales continuarían con un crecimiento moderado, los nuevos suelos residenciales absorberían el grueso del crecimiento demográfico en los próximos años. Si se calcula un crecimiento demográfico similar y progresivo respecto al observado durante los últimos años, un número medio de viviendas principales semejante al actual (70,3\%) para las nuevas urbanizaciones, y un valor medio de habitantes en estas viviendas de 2,78 , podrían aparecer importantes aglomeraciones como Las Cañadas-El Tite en Elda (13.038 habitantes), Mayorazgos (7.650) y Betíes (7.076) en Monóvar, Serreta Golf en Novelda (4.999) o El Rincón (2.072) y La Solana (2.005) en Hondón de las Nieves. En conjunto, lo que se observa es una dinámica aparición de nuevos núcleos que, desde los principales núcleos de población, se extiende cada vez con más intensidad hacia las ciudades de tamaño reducido y los pueblos rurales ubicados al oeste de la comarca.

Finalmente, como elementos articuladores de los usos primarios del territorio y los asentamientos de población, es preciso hacer referencia a la disposición de los canales de conexión. De los planes estatales y autonómicos de infraestructuras, se desprende la intención de hacer accesibles todos los espacios de la comarca a través de viales de gran capacidad o de la mejora de las conexiones existentes. El proyecto de ampliación de la autovía Madrid-Alicante, el eje propuesto entre Elda-Petrer y Pinoso (autovía del mármol) o el trazado de la autovía Yecla-Santomera (cuyo itinerario discurre por el término municipal de Pinoso), generarán una ampliación de las comunicaciones entre el norte-sur y este-oeste de la comarca, así como una mayor permeabilidad desde el exterior. Por otra parte, el trazado 
del tren de alta velocidad provocará una importante transformación del corredor central de comunicaciones de la comarca, con un fuerte impacto territorial derivado del efecto barrera de la nueva infraestructura, que no se corresponderá con una influencia funcional o socioeconómica (al carecer de paradas en los municipios de la comarca).

En síntesis, el modelo territorial resultado de la simulación de los diferentes planes y proyectos de actuación, ofrece un escenario futuro de difusas e impredecibles consecuencias. La magnitud de las actuaciones revela el modo de operar de las administraciones locales, que actúan como si el territorio no tuviese límites y no importase la posición de los municipios vecinos. Las propuestas municipales no advierten la capacidad de acogida del territorio y no justifican la verdadera demanda de viviendas, suelo industrial, instalaciones deportivas y terciarias o vías de comunicación. En un ambiente de euforia constructora, los ayuntamientos han planteado individualmente su crecimiento territorial deseado y no han contado con unas directrices supramunicipales que adviertan de los posibles riesgos que implica. Una imagen territorial futura como la planteada debería hacer reflexionar sobre las consecuencias de este modelo de desarrollo, pues sus resultados económicos, ambientales y sociales, podrían ser verdaderamente caóticos y catastróficos.

\section{Territorio y urbanismo: testimonio espacial de la crisis en el Medio Vinalopó}

\subsection{Los efectos del modelo de planificación territorial}

El modelo de planificación vigente ha puesto de relieve una serie de inconvenientes derivadas del fondo y de las formas con las que se está aplicando. En el fondo, porque se trata de un modelo de crecimiento disperso y de ocupación desordenada y depredadora del territorio, que se aleja de las directrices europeas que apuestan por una gestión prudente del territorio y de los recursos. Y en las formas, por las evidencias de colusión de intereses, de confusión entre lo público y lo privado, de utilización de información privilegiada para especular, de corrupción en definitiva (Boira y otros, 2005: 314). Además, la euforia provocada en un contexto de bonanza económica, en la cual toda inversión destinada a la urbanización se consideraba una apuesta sobre seguro, ha terminado generando el efecto contrario. Las negativas consecuencias de un periodo caracterizado por la expansión urbanizadora se han contagiado entre las empresas privadas, las administraciones públicas y, finalmente, ha afectado al conjunto de la sociedad a través de los recortes en gasto social y el aumento de la desocupación. Por todo ello, considerando los perjuicios económicos, ambientales y sociales derivados del boom inmobiliario, es posible afirmar que las formas de uso y gestión del territorio se convierten en el testimonio espacial de la actual crisis.

Desde el punto de vista económico, en el Medio Vinalopó, los efectos de esta situación están siendo particularmente devastadores por dos cuestiones. En primer lugar, por la desinversión experimentada en los sectores industriales tradicionales, ya que buena parte de los recursos se han ido redestinado hacia fines urbanísticos. Los sectores industriales tradicionales, especialmente el calzado (sumido en una crisis casi estructural), sin la atención pública que tuvo en otro tiempo, se ha ido descapitalizando, perdiendo rentabilidad para el empresariado y reduciendo progresivamente su mano de obra. Mientras, la industria de la piedra natural, tras el parón de la construcción, está sufriendo una pérdida de demanda que se traduce igualmente en una reducción de la actividad y en un aumento de la desocupación. En segundo lugar, la caída de la actividad constructora también se ha llevado por delante miles de puestos de trabajo de empleados sin otro tipo de especialización. Así, al desempleo generado por las actividades tradicionales, ya de por sí elevado, cabe ahora incorporar los 
nuevos parados del sector de la construcción. En conjunto, en $2009^{8}$ se registran 22.188 desempleados, lo que supone un $305 \%$ más respecto al año 2000, y un $242 \%$ respecto a 2005. La nota más destacada es que, a diferencia de lo que ocurre en el ámbito regional y subregional, una mayoría de los desocupados proceden de la actividad industrial (43\%), mientras que un $14 \%$ procede del sector de la construcción ${ }^{9}$. En cualquier caso, el aumento del paro desde el año 2000, en valores absolutos, está siendo espectacular en municipios como Elda, Petrer o Novelda, donde se registran respectivamente 4.135, 3.663 y 2.117 nuevos desocupados. A pesar de todo, no parece haber una alternativa que en el corto plazo permita solucionar esta cuestión.

De otro lado, el modelo de crecimiento urbanístico ha provocado un fuerte endeudamiento de algunos municipios, derivado de los costes de un proceso que sólo es rentable unos pocos años. Recuperando algunas ideas del arquitecto García Bellido, «los beneficios del desarrollo actual sólo duran mientras se cobran las licencias, vienen los constructores y se instalan los nuevos pobladores; pero se sumen en el déficit creciente cuando estos movimientos se acaban y hay que dotar de servicios, equipamientos, empleos fijos, suministros, etc. y renovar las infraestructuras en el mastodóntico suelo urbano así extensivamente creado, insostenible y costoso» (García, 2004: 7). Esto puede llegar a explicar que Monforte del Cid, uno de los municipios más dinámicos desde el punto de vista urbanístico, haya alcanzado la mayor deuda por habitante de la provincia ${ }^{10}$.

Pero el último periodo de expansión urbanizadora ha tenido otro tipo de costes difícilmente mesurables en términos monetarios. El dinámico proceso de venta y consumo artificial de suelo está teniendo repercusiones sobre el territorio y el paisaje que pueden desembocar en graves e irreversibles daños en el futuro. La transformación masiva de determinados espacios o la ocupación de lugares especialmente frágiles para dar cabida a las nuevas urbanizaciones o áreas industriales está generando una fuerte hipoteca de territorio y paisaje. En municipios como Monforte del Cid, Elda, Hondón de las Nieves y Hondón de los Frailes, de desarrollar las actuaciones previstas, se habrá ocupado todo el suelo disponible, en algunos casos a costa de la recalificación de espacios de interés natural y cultural (es el caso del sector Las Cañadas-El Tite en Elda). La falsa idea de que el territorio es ilimitado y lo puede acoger todo está muy extendida entre los políticos y técnicos municipales, como así manifiestan las palabras del arquitecto municipal de Monóvar en 2005, quien señalaba que «el término municipal de Monóvar es uno de los más extensos de la provincia y su núcleo urbano está situado en un extremo, con lo cual aún hay mucho territorio virgen ${ }^{11} \gg$. De este modo se ha ido justificando un tipo de planificación que se pretende extensiva y altamente consumidora de territorio y paisaje, especialmente el de calidad. Así, enclaves como La Boticaria, Mayorazgos o Betíes en Monóvar, Velaire en La Romana, La Solana en Algueña, El Rincón en Hondón de las Nieves, La Serreta en Novelda o Font del Llop en Monforte del Cid, han sido elegidos por las empresas promotoras para implantar zonas residenciales, próximas a espacios naturales, en paisajes rurales y que ofrecen el atractivo de las vistas y la tranquilidad a los potenciales compradores. Este modelo de crecimiento urbanístico, caracterizado por la implantación de grandes piezas de suelo urbano fragmentadas y dispersas en el territorio, conectadas a través de viales de gran capacidad, confirmaría una tendencia de artificialización de suelo

\footnotetext{
8 Todos los datos de desempleo están referidos a fecha de 31 de marzo. Fuente: www.ive.es

9 En la provincia de Alicante, el $21 \%$ de los parados corresponden a la industria y el $22 \%$ a la construcción. En la Comunidad Valenciana los datos son del 20 y $21 \%$ respectivamente.

10 Diario Información (1-4-2009): «Monforte del Cid tiene la mayor deuda por habitante de la provincia».

11 Diario El Mundo (1-12-2005): «El nuevo PGOU prevé que Monóvar crezca de 13.000 a 57.000 habitantes en 20 años».
} 
que ha venido en aumento desde el año 2000, pasando de un 5,6\% (4.430 hectáreas) de superficies artificiales, a un 6,9\% en 2007 y a un hipotético $11,5 \%$ en la próxima década.

Este modelo de uso y gestión del territorio, ha merecido además una creciente preocupación ciudadana, que se refleja en el aumento de voces que exigen nuevas formas de entender el territorio y nuevos canales de planificación territorial. Cada vez han sido más los ciudadanos que, de modo individual, o a través de colectivos como Asociación Nueva Cultura del Territorio, Plataforma por un urbanismo sostenible o las diferentes asociaciones vecinales y ecologistas locales, han denunciado las injusticias del modelo de planificación y han propuesto alternativas al mismo. Especialmente dinámicas han sido las asociaciones de vecinos de Hondón de los Frailes, Hondón de las Nieves o Monóvar ${ }^{12}$ en la exigencia de un mayor respeto por las propiedades privadas en los proyectos propuestos. Plataformas como Tren sí-AVE no o Zona 15 No, exigiendo alternativas ambientalmente más racionales a los proyectos del tren de alta velocidad y el Plan Eólico Valenciano. Asociaciones como Mosaico (Elda), Ecologia i Pau (Novelda) o El Tramvia ${ }^{\circ} 2$ (La Romana), reclamando más transparencia e información en las fases de elaboración de políticas territoriales. En conjunto, se identifican multitud de colectivos cívicos que rebelan una falta de confianza en las instituciones encargadas de la política territorial, que muestran una creciente preocupación por la calidad, los recursos, la seguridad y la identidad del lugar donde vive, y, en definitiva, que manifiestan las carencias de una política territorial que suele estar mal diseñada y casi siempre mal explicada (Nel·lo, 2003: 12).

Ante estos problemas, se plantean multitud de retos que pasan por replantear el modelo de planificación territorial y urbanística vigente. Son necesarios nuevos principios que relacionen al territorio con la coherencia económica, social y ambiental, y que por tanto planteen el territorio desde un punto de vista integral, que afecta e incluye a todos los sectores económicos y toda la población.

\subsection{Los límites de la planificación y el necesario cambio de enfoque}

El modelo de planificación territorial valenciano, basado en el individualismo del municipio y la ausencia de directrices regionales y supramunicipales se ha agotado. La crisis económica, que es también una crisis del y con manifestaciones en el territorio y la política territorial, ha revelado la existencia de multitud de conflictos en el uso y gestión del suelo, en el modelo tecnocrático de planificación, en la falta de canales de consenso y participación en la política territorial. En definitiva, una falta de cultura territorial con nefastas consecuencias ambientales, económicas y sociales.

Son necesarias alternativas que aboguen forzosamente por un control de los procesos urbanizadores y el abuso de los recursos territoriales. Son necesarios nuevos enfoques de planificación territorial estratégica, que entiendan integralmente el territorio y todos los sectores y a la población que sobre éste se desarrollan. Se precisan, igualmente, otros modos de hacer política territorial, basados en el interés de las administraciones públicas y las empresas privadas, pero también de todos los actores locales y especialmente la ciudadanía. Tal vez sea este el momento de revisar y dar validez a algunas de las ideas que surgen desde colectivos profesionales y cívicos. El Manifiesto por una nueva cultura del territorio, el Manifiesto por una democracia del territorio ${ }^{13}$ o las 40 propuestas por una

12 Estos colectivos tuvieron un destacado protagonismo en la elaboración de los informes sobre el impacto de la urbanización extensiva en España, presentados por los eurodiputados Fourtou (2005), Cashman y Libicki (2007) y Auken (2009) en el Parlamento Europeo.

13 http://www.nosevende.org/MANIFIESTO.pdf 
vivienda digna ${ }^{14}$, pueden contribuir a elaborar una hoja de ruta que permita buscar soluciones a la crisis desde una planificación territorial sensata y coherente.

A pasos pequeños y lentos, parece que el cambio de enfoque puede ser posible. La Estrategia Territorial Valenciana avanza y algunos de los PAT más relevantes están muy desarrollados, como por ejemplo, el PAT de protección de las huertas de Valencia. A nivel supramunicipal, parece además haber una mayor voluntad por coordinar las políticas territoriales desde perspectivas más amplias, y la idea de áreas funcionales se recupera con el objetivo de diagnosticar y proponer sobre ámbitos comarcales. Así por ejemplo, el 30 de marzo de 2010 se presentaron en Elda los objetivos de la Estrategia respecto al área funcional del Vinalopó, que incluye las comarcas del Alto y Medio Vinalopó. No debería ser esta una propuesta de apariencia y de simples formalismos, sino un proceso efectivo, en el cual participasen activamente administraciones locales y ciudadanía, aunque se antoja ésta como una cuestión difícil.

La crisis debe ser aprovechada para provocar un cambio de modelo de crecimiento urbanístico y planificación territorial, diferentes a los que han conducido a la situación actual. Por tanto, son necesarios nuevos criterios como el enfoque supramunicipal, la persuasión de los individualismos localistas, la coordinación y la cultura del consenso, la participación ciudadana, el cambio de modelo productivo y el cambio de cultura territorial. Para ello es imprescindible la voluntad de los poderes políticos y el apoyo de numerosos actores, si de lo que se pretende es cambiar el rumbo de la crisis.

\section{Conclusiones}

La planificación territorial y urbanística en la Comunidad Valenciana ha favorecido la venta y consumo artificial de suelo, que ha sido además el soporte de valor que ha justificado la bonanza económica de los últimos años. Esta situación, que en un principio parecía ser exclusiva de las áreas turísticas litorales, se extiende y se hace cada vez más intensa en espacios próximos del interior, cuya dinámica, modelo productivo y trayectoria urbanística poco tenía que ver con lo sucedido en el litoral. La comarca del Medio Vinalopó se incorpora a este proceso en la segunda etapa del último periodo de expansión urbanizadora, y en ella se detecta una euforia y un dinamismo constructor hasta el momento insólito en la mayor parte de sus municipios.

Durante los últimos años, se enuncian e inician multitud de proyectos de actuación en el territorio que incorporan miles de viviendas, varios campos de golf, millones de metros cuadrados para nuevo suelo industrial y decenas de kilómetros de nuevas infraestructuras de transporte de gran capacidad. Una visión conjunta de todas estas propuestas, simuladas en un horizonte temporal en el medio plazo, ofrece un escenario territorial futuro de alarmantes e impredecibles consecuencias. Como resultado del individualismo localista en la planificación territorial, los crecimientos se producen de forma desmesurada en cada municipio, con actuaciones que cuestionan los límites del territorio.

La actual situación de crisis en algunos casos ha frenado determinados crecimientos urbanísticos. No obstante, el modelo hasta el momento vigente ha provocado importantes secuelas sobre el territorio y el paisaje, la base económica y la estructura de empleo de los municipios comarcales, y ha bipolarizado como nunca antes había ocurrido a la clase política local y la sociedad civil. En definitiva, una crisis que tiene sus causas y sus consecuencias en el territorio, y que por tanto debe buscar soluciones desde el mismo.

14 http://www.viviendadigna.org/?reivindicaciones.php 
Por tanto, la Comunidad Valenciana precisa un cambio de enfoque en su planificación territorial, que incorpore objetivos de racionalidad, coherencia, consenso y gestión participada. Esto debe acompañarse de un cambio del modelo productivo que erradique los factores que han conducido a la situación de crisis actual. Es precisa la intervención de los poderes políticos y de todos los actores sociales en un reto compartido que contribuya a convertir en oportunidades las consecuencias de la crisis.

\section{Bibliografía}

BOIRA, J.V.; ROMERO, J. y SORRIBES, J. (2005): «¿Qué modelo territorial? Apuntes para un debate inaplazable», en ROMERO, J. y ALBEROLA, M. (Coords.): Los límites del territorio. El País Valenciano en la encrucijada. Universitat de València. Valencia, pp. 309-325.

BURRIEL, E. (2009a): «Los límites del planeamiento urbanístico municipal. El ejemplo valenciano», en Documents d'Anàlisi Geogràfica, no 54 , pp. 33-54.

BURRIEL, E. (2009b): «La planificación territorial en la Comunidad Valenciana (1986-2009)», en Scripta Nova. Revista Electrónica de Geografía y Ciencias sociales, vol. XIII, nº 306. (http:// www.ub.es/geocrit/sn/sn-306.htm)

DÍAZ, F. \& LOURÉS, M.L. (2008): «La globalización de los mercados inmobiliarios: su impacto sobre la Costa Blanca», en Ciudad y Territorio: Estudios Territoriales, XL (155), pp. 77-92.

FERNÁNDEZ, R. (2006): El tsunami urbanizador español y mundial. Sobre sus causas y repercusiones devastadoras, y la necesidad de prepararse para el previsible estallido de la burbuja inmobiliaria. Virus Editorial. Barcelona, 88 pp.

GAJA, F. (2008); «El tsunami urbanizador en el litoral mediterráneo. El ciclo de hiperproducción inmobiliaria 1996-2006», en Scripta Nova. Revista Electrónica de Geografía y Ciencias Sociales, vol. XII, núm. 270 (66). (http://www.ub.es/geocrit/sn/sn-270/sn-270-66.htm)

GARCÍA, J. (2004): «Y, cuando se acabe el suelo del municipio... ¿qué hacer?», en Ciudad y Territorio: Estudios Territoriales, XXXVI (139), pp. 5-13.

GIMÉNEZ, P. y DÍEZ, D. (2009): «Contexto rural y crecimiento urbanístico en el traspaís de Benidorm: un análisis crítico del actual modelo de desarrollo territorial», en FERIA y otros (Eds.): Territorios, sociedades y políticas. Universidad Pablo de Olavide. Sevilla, pp. 275-285.

GÓMEZ, D. (2008): Ordenación territorial. Mundi-Prensa. Madrid, 766 pp.

LLEÓ, J. (1979): «La actitud prospectiva en la ordenación del territorio», en Revista de Obras Públicas, septiembre 1979, pp. 729-738.

MATA, R. (2007): Auge inmobiliario y evolución de los usos de suelo en España. Por una nueva cultura del territorio. Universidad Autónoma de Madrid, apertura del curso académico 20072008, lección inaugural. 67 pp.

NAVARRO, J.R. \& ORTUÑO, A. (2008): Estudio sobre el impacto territorial de los campos de golf y operaciones asociadas en el Levante español. Ciudades privadas: de la utopía al negocio inmobiliario. Universidad de Alicante, Dpto. Geografía Humana. Inédito, 83 pp.

NEL.LO, O. (Ed.) (2003): Aquí, no! Els conflictes territorials a Catalunya. Empúries. Barcelona, $461 \mathrm{pp}$.

OBSERVATORIO DE LA SOSTENIBILIDAD EN ESPAÑA (2006): Cambios de ocupación del suelo en España. Implicaciones para la sostenibilidad. OSE. Madrid, $485 \mathrm{pp}$.

RICO, A. y HERNÁNDEZ, M. (2008): «Ordenación del territorio, escasez de recursos hídricos, competencia de usos e intensificación de las demandas urbano-turísticas en la Comunidad Valenciana», en Documents d'Anàlisi Geogràfica, no ${ }^{\circ}$ 1, pp. 79-109.

ROMERO, J. (2009): Geopolítica y gobierno del territorio en España. Tirant lo Blanch. Valencia, $273 \mathrm{pp}$.

VV.AA. (2009): Territorio, urbanismo y crisis. (http://nuevaculturadelterritorio.files.wordpress. com/2009/02/territorio_urbanismo_y_crisis.pdf) 